\title{
The MUon Scattering Experiment (MUSE) at the Paul Scherrer Institute
}

\author{
Steffen Strauch*tfor the MUSE Collaboration \\ University of South Carolina \\ E-mail: strauchesc.edu
}

\begin{abstract}
While consistent results for the charge radius of the proton have been extracted from elastic electron-scattering data and through the spectroscopy of atomic hydrogen, high-precision studies of muonic hydrogen found notably smaller values for the charge radius. This so-called protonradius puzzle raises questions ranging from experimental and methodological issues to physics beyond the standard model. The puzzle certainly calls for new measurements. The MUon proton Scattering Experiment (MUSE) at the Paul Scherrer Institute (PSI) will provide elastic scattering data off the proton with electron and muon beams of positive and negative charge in a fourmomentum-transfer range from 0.002 to $0.08 \mathrm{GeV}^{2} / c^{2}$. Each of the four sets of data will allow the extraction of the proton charge radius; in combination, the data test possible differences of the electron and muon interactions and additionally two-photon exchange effects. The experiment is presently being commissioned at PSI. An overview of the experiment will be presented.
\end{abstract}

The 20th International Workshop on Neutrinos (NuFact2018)

12-18 August 2018

Blacksburg, Virginia

\footnotetext{
*Speaker.

${ }^{\dagger}$ Supported in parts by the U.S. National Science Foundation: NSF PHY-1505615
} 
Recent high-precision measurements of the Lamb shift in the muonic hydrogen atom [1,2] obtained a significantly smaller value of the proton charge radius compared to values based on, both, electronic hydrogen spectroscopy and on electron scattering [3]. In the scattering experiment the radius is found from the slope of the electric form factor, $r_{p}^{2}=-6 \hbar^{2} d G_{E}^{p} /\left.d Q^{2}\right|_{Q^{2}=0}$, where $Q^{2}$ is the four-momentum-transfer to the proton. A recent review article [4] discusses many suggested solutions to the puzzle. First, there may be problems with the experiment, including underestimation of uncertainties, difficulties in fitting the slope of the electric form factor for the radius extraction in case of scattering experiments, or issues in QED calculations in the analysis of spectroscopy data. Second, novel hadronic physics may be important for $\mu p$ but not for ep systems. Finally, the discrepancy may be an indication of the violation of $\mu / e$ universality and hint at physics beyond the standard model. Several ideas have been ruled out, and none have gained universal acceptance.

Since then, several pieces of the puzzle are coming together: New hydrogen spectroscopy measurements have been obtained, but with conflicting results, supporting the case for both, a smaller [5] and larger [6] proton charge radius. New electron scattering experiments will improve on the precision of the form factor extraction and reach lower values of $Q^{2}$, e.g., the proton radius (PRad) experiment at JLab Hall B [7] or the Initial State Radiation (ISR) experiment at MAMI [8]. What is missing, however, are high-precision scattering data with muons. This void is filled with the Muon Scattering Experiment (MUSE) [9] at the Paul Scherrer Institute (PSI). This unique experiment is the only one, which will directly compare $e p$ and $\mu p$ in an elastic scattering experiment. The MUSE experiment will measure $e^{ \pm} p$ and $\mu^{ \pm} p$ elastic-scattering cross sections in a $Q^{2}$ range between 0.002 and $0.08 \mathrm{GeV}^{2} / c^{2}$ in overlapping kinematic settings with beam momenta of 115,153 , and $210 \mathrm{MeV} / c$. Measurements with both charges for the lepton allow for studies of possible two-photon exchange mechanisms. The use of both $e$ and $\mu$ beams provides for a direct test of lepton-type-dependent effects. In the absence of inelastic reactions off the proton, the determination of the lepton scattering angle at a given beam momentum completely determines the kinematics of the reaction. A magnetic spectrometer to measure the scattered particle momentum is not needed. A schematic diagram of the setup is shown in Fig. 1. The low beam flux requires large-acceptance detectors. Electrons, muons, and pions from the secondary $\pi \mathrm{M} 1$ beam line are timed relative to the radio-frequency of the accelerator and identified in the beam hodoscope. Electrons and muons are then tracked with a set of GEM detectors into the liquid-hydrogen target inside of the target chamber. The GEM detectors have achieved a position resolution of $70 \mu \mathrm{m}$. A veto detector in front of the target chamber reduces trigger rate from background tracks. The straw-tube tracker provides high-resolution and high-efficiency tracking of the scattered particles from the target. The high-resolution, $<60 \mathrm{ps,} \mathrm{scattered-particle} \mathrm{scintillators} \mathrm{serve} \mathrm{in} \mathrm{the} \mathrm{event} \mathrm{trigger} \mathrm{and}$ provide timing for the rejection of muon-decay background. The beam monitor, downstream of the target, helps to measure beam properties and to suppress background from Møller and Bhabha scattering. Together with the beam hodoscope it also allows for a precise muon- and pion-momentum determination using the time-of-flight technique.

The experiment aims for systematic uncertainties of the elastic cross sections below $0.5 \%$. The sensitivity to differences in the extracted proton radius from $e$ and $\mu$ scattering data is expected to be $\pm 0.005 \mathrm{fm}$. A detailed discussion of the experiment is given in the MUSE Technical Design Report [9]. The setup and the detectors are mostly commissioned and await production runs in 2019. 


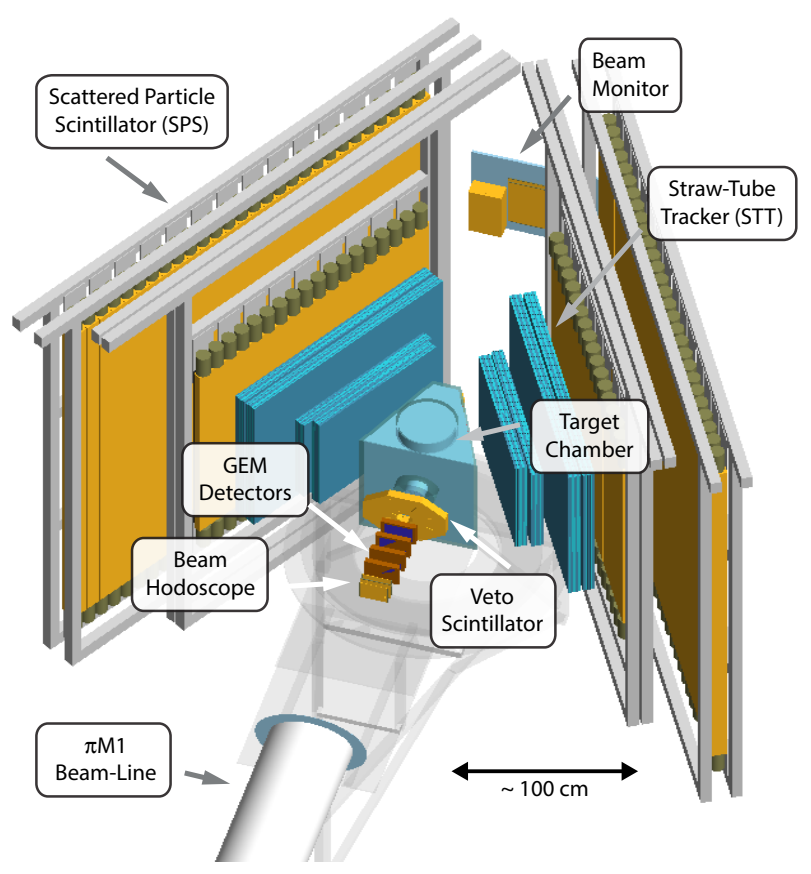

Figure 1: Geant4-based schematic view of the detector setup for the MUSE experiment at the $\pi \mathrm{M} 1$ beamline at the Paul Scherrer Institute including beam-line instrumentation and scattered particle detectors.

\section{References}

[1] R. Pohl, A. Antognini, F. Nez, F. Amaro, F. Biraben et al., The size of the proton, Nature 466 (2010) 213.

[2] A. Antognini, F. Nez, K. Schuhmann, F. Amaro, F. Biraben et al., Proton Structure from the Measurement of 2S-2P Transition Frequencies of Muonic Hydrogen, Science 339 (2013) 417.

[3] P. J. Mohr, D. B. Newell and B. N. Taylor, CODATA Recommended Values of the Fundamental Physical Constants: 2014, Rev. Mod. Phys. 88 (2016) 035009 [1507.07956].

[4] R. Pohl, R. Gilman, G. Miller and K. Pachucki, Muonic hydrogen and the proton radius puzzle, Ann. Rev. Nucl. Part. Sci. 63 (2013) 175 [1301. 0905].

[5] A. Beyer, L. Maisenbacher, A. Matveev, R. Pohl, K. Khabarova, A. Grinin et al., The rydberg constant and proton size from atomic hydrogen, Science 358 (2017) 79.

[6] H. Fleurbaey, S. Galtier, S. Thomas, M. Bonnaud, L. Julien, F. Biraben et al., New Measurement of the 1S - 3S Transition Frequency of Hydrogen: Contribution to the Proton Charge Radius Puzzle, Phys. Rev. Lett. 120 (2018) 183001 [1801.08816].

[7] PRAD collaboration, A. H. Gasparian, The New Proton Radius Experiment at Jefferson Lab, JPS Conf. Proc. 13 (2017) 020052.

[8] M. Mihovilovič et al., First measurement of proton's charge form factor at very low $Q^{2}$ with initial state radiation, Phys. Lett. B771 (2017) 194 [1612.06707].

[9] MUSE collaboration, R. Gilman et al., Technical Design Report for the Paul Scherrer Institute Experiment R-12-01.1: Studying the Proton "Radius" Puzzle with $\mu$ p Elastic Scattering, 1709.09753. 